\title{
Validação de escalas para avaliação do amor patológico
}

\author{
Validation of scales for pathological love assessment
}

\author{
Marina Perito Berti¹, Monica Levit Zilberman¹, Eglacy Cristina Sophia ${ }^{1}$, Clarice Gorenstein ${ }^{1}$, Ana Paula Pereira ${ }^{1}$, \\ Andréa Lorena1, Cidália Mello², Táki Athanássios Cordás¹, Hermano Tavares ${ }^{1}$
}

1 Instituto de Psiquiatria do Hospital das Clínicas da Faculdade de Medicina da Universidade de São Paulo (IPq/HCFMUSP).

2 Departamento Municipal de Saúde de Miracatu/SP.

Recebido: 10/7/2010 - Aceito: 21/10/2010

\section{Resumo}

Contexto: Diversas metodologias foram desenvolvidas para validar instrumentos psicométricos. No entanto, na literatura científica, há escassez de instrumentos para avaliar os relacionamentos amorosos. Objetivo: O presente trabalho tem como objetivo validar para o português o conteúdo dos instrumentos autoaplicáveis Love Attitudes Scale (Escala de Atitudes do Amor - Hendrick et al., 1998), Relationship Assessment Scale (Escala de Avaliação do Relacionamento - Hendrick, 1988) e Adult Attachment Types (Tipos de Apego do Adulto - Hazan et al., 1987), levando em consideração o contexto cultural brasileiro e, consequentemente, possibilitando que o estudo do amor patológico seja viável na população brasileira. Métodos: Tal processo envolveu quatro etapas: (1) tradução para o português, (2) retrotradução, (3) apresentação da versão corrigida para profissionais de saúde mental e estudantes universitários e (4) aplicação da versão final em amostra da população-alvo utilizando uma escala analógico-visual. Resultados: As escalas foram facilmente compreendidas e discriminaram significativamente indivíduos com amor patológico de voluntários saudáveis. Conclusão: Nossos resultados mostram que a versão brasileira desses instrumentos é válida e pode ser usada em investigações envolvendo relacionamentos amorosos.

Berti MP, et al. / Rev Psiq Clín. 2011;38(4):135-8

Palavras-chave: Amor, escalas, comportamento compulsivo, validação, tradução.

\begin{abstract}
Background: Several methodologies were developed to validate psychometric instruments. However, there are, in the scientific literature, a lack of instruments to evaluate romantic relationship. Objective: The present work aims at validating into Portuguese the content of the self-report Love Attitudes Scale (Escala de Atitudes do Amor - Hendrick et al., 1998), the Relationship Assessment Scale (Escala de Avaliação do Relacionamento - Hendrick, 1988), and the Adult Attachment Types (Tipos de Apego do Adulto - Hazan et al., 1987), taking the Brazilian cultural context into consideration, thus rendering the study of pathological love in the Brazilian population feasible. Methods: Such process involved four stages: (1) translation into Portuguese, (2) back translation, (3) presentation of the translated version to mental health professionals and university students, and (4) application of the final version in a sample of the target population using a rating scale. Results: The scales were easily understood and significantly discriminated individuals with pathological love and healthy volunteers. Discussion: Our results show that the Brazilian version of these instruments is valid and may be used in investigations involving romantic relationships.
\end{abstract}

Berti MP, et al. / Rev Psiq Clín. 2011;38(4):135-8

Keywords: Love, scales, compulsive behavior, validation, translating.

\section{Introdução}

O amor patológico é caracterizado pelo comportamento de prestar atenção e cuidados ao parceiro de maneira repetitiva e desprovida de controle em uma relação amorosa, sendo essa conduta prioritária para o indivíduo em detrimento de outros interesses antes valorizados ${ }^{1-3}$.

O amor patológico pode ocorrer associado a outros transtornos psiquiátricos, a sintomas depressivos e ansiosos primários ${ }^{1,4-6}$ ou isoladamente, em personalidade vulnerável 7,8, com baixa autoestima ${ }^{7,9,10}$ e sentimentos de rejeição, abandono e raiva ${ }^{6,7,11}$. Fatores familiares também podem estar associados ao seu desenvolvimento, como pais usuários de álcool ou drogas e histórico de negligência física e/ou emocional durante a infância ${ }^{4}$.

O diagnóstico de transtornos psiquiátricos é feito com base em sintomas e síndromes clínicas, visto que não existem, até o momento, marcadores biológicos ou outras representações mensuráveis objetivas para esse fim. Nos anos 1960 e 1970, foram desenvolvidos estudos que evidenciaram grandes variações que ocorrem nos diagnósticos clínicos quando diferentes psiquiatras avaliam um mesmo grupo de indivíduos, em virtude das diferenças de critérios diagnósticos que cada profissional adota e à maneira como cada um deles obtém informações dos indivíduos que estão sendo avaliados. A partir de então, foi desenvolvida uma série de entrevistas psiquiátricas padronizadas, ou instrumentos, visando aumentar a concordância entre investigadores, além de possibilitar comparações de resultados. Atualmente, a utilização desses instrumentos tornou-se obrigatória em qualquer pesquisa na área de psiquiatria ${ }^{12}$.

O presente trabalho tem como objetivo validar para o português três dos instrumentos utilizados em pesquisas relacionadas ao amor patológico: 1) Love Attitudes Scale ${ }^{13}$ (Escala de Atitudes do Amor): 24 itens, divididos em seis estilos de amor, resultando em escores que indicam o estilo predominante do indivíduo, como proposto por Lee ${ }^{14}$ : Eros (itens 1 a 4: atração física; comprometimento mútuo; seguro; não inclui possessividade), Ludus (itens 5 a 8: sedução; liberdade sexual; encara o amor como um jogo), Estorge (itens 9 a 12: afinidade; surge, geralmente, a partir de uma longa amizade), Pragma (itens 13 a 16: avaliação dos pretendentes antes de se envolver; encara o amor como uma questão prática), Mania (itens 17 a 20: obsessão; ciúme; indivíduo se sente forçado a atrair continuamente a atenção do parceiro) e Ágape (itens 21 a 24: proteção e cuidado; apoio incondicional; altruísmo);2) Relationship Assessment Scale ${ }^{15}$ (Escala de Avaliação do Relacionamento): sete itens, resultando em um escore que varia de 7 a 35 e avalia a satisfação no relacionamento; apresenta boa consistência interna (alfa $=0,86)$ e validade preditiva, distinguindo significativamente os casais que permanecem unidos daqueles que rompem; 3) Adult Attachment Types ${ }^{16}$ (Tipos de Ape- 
go do Adulto): três afirmativas que representam tipos de apego do indivíduo em relação ao parceiro, baseando-se no apego materno vivenciado na infância. A teoria do apego foi proposta por Bolwby ${ }^{17}$ e, posteriormente, classificada por Ainsworth et al. ${ }^{18} \mathrm{em}$ : apego seguro (mãe disponível às necessidades da criança: indivíduo apto a vivenciar um amor saudável), apego ansioso com evitação (rejeição da mãe quando o indivíduo a procurava para obter proteção: indivíduo tenta viver de maneira autossuficiente) e apego ansioso/resistente ou ambivalente (mãe esteve disponível em algumas situações e não em outras: indivíduo sofre de ansiedade de separação na vida adulta).

Todos esses estudos foram desenvolvidos em outros países e culturas. Tendo em vista que instrumentos estrangeiros recebem forte influência do contexto cultural em que foram desenvolvidos, nem sempre a colocação de um conceito pode ser reproduzida de uma cultura para outra ${ }^{19}$. É necessário, portanto, que os instrumentos de avaliação citados sejam validados para o português e para a realidade brasileira, a fim de que sejam sensíveis ao nosso contexto cultural.

\section{Métodos}

1) Tradução dos instrumentos originais para o português, realizada por um tradutor/psicólogo. 2) Retrotradução feita por outro tradutor profissional. 3) Apresentação das versões corrigidas a cinco profissionais de saúde mental e cinco universitários para que sugerissem modificações quando a linguagem não lhes parecesse adequada. 4) Aplicação das versões finais (juntamente com uma escala verbal numérica de cinco pontos, utilizada para avaliar a compreensão dos instrumentos) a 39 indivíduos: 19 recrutados entre pacientes com diagnóstico de amor patológico do Ambulatório Integrado dos Transtornos do Impulso (Amiti) e 20 entre voluntários saudáveis, sem patologia psiquiátrica, conforme avaliação pelo SCID ${ }^{20} .5$ ) Análise estatística (nível de significância: $\mathrm{p}=0,05$ ) incluindo: testes de normalidade para cada item das escalas (Skewness); comparação demográfica entre as amostras ( $t$ de Student: variáveis contínuas/ distribuição normal; qui-quadrado: variáveis categoriais; Mann-Whitney: variáveis ordinais/contínuas não paramétricas); análise de covariância (ANCOVA) para comparação das escalas entre os grupos, considerando dados demográficos significativamente diferentes como covariáveis; correlação de Spearman (variáveis não paramétrica) e Pearson (variáveis normais) para verificar associação entre variáveis (escolaridade, pontuação, compreensão); consistência interna (alfa de Cronbach); regressão logística incluindo estilos de amor (Escala de Atitudes do Amor) significantes a 10\% e dados demográficos significativamente diferentes.

\section{Resultados}

A análise estatística mostrou que os grupos são homogêneos, exceto pela escolaridade (Tabela 1). Indivíduos com amor patológico têm escolaridade significativamente maior do que os voluntários saudáveis.

\section{Escala de Atitudes do Amor}

Houve diferenças significativas de estilos de amor entre os grupos, exceto por Ludus e Pragma (Tabela 1). O grupo saudável foi caracterizado por Eros (70\%), enquanto o grupo com amor patológico, por Mania $(74 \%)$.

ANCOVA revelou que Eros foi influenciado pela escolaridade $(\mathrm{R}=0,495 ; \mathrm{p}=0,001)$.

A regressão logística foi utilizada para verificar os estilos que distinguiram os grupos ao nível de significância de 10\%, incluindo, portanto, Eros, Estorge, Mania e Ludus, controlados para a escolaridade (Ágape não foi incluído por causa de sua significante correlação com Mania: $\mathrm{R}=0,544 ; \mathrm{p}<0,001)$. O modelo final foi significativo $\left(\mathrm{R}^{2}=0,871 ; \chi^{2}=41,27 ; \mathrm{p}<0,001\right)$ e incluiu Mania (e, portanto, Ágape) e Eros.

As médias de compreensão ficaram entre 3,8 e 5,0. Houve diferenças entre os grupos no entendimento dos itens $1,2,4,5$ e 8 , porém os voluntários saudáveis apresentaram melhor entendimento. Foram feitos, então, testes de correlação de Spearman para analisar a correla- ção entre pontuação e compreensão dos itens 1, 2, 4, 5 e 8 (separadamente para cada grupo e para o total da amostra), verificando-se que não houve interferência da compreensão desses itens na pontuação.

A consistência interna da escala foi avaliada para cada subescala que representa os diferentes estilos de amor (itens 1-4: Eros; itens 5-8: Ludus; itens 9-12: Estorge; itens 13-16: Pragma; itens 17-20: Mania; itens 21-24: Ágape). Eros obteve consistência razoável (alfa $=0,741$ ), porém a questão 4 ("Meu parceiro se encaixa nos meus padrões ideais de beleza física.") não contribuiu para o conceito. Ludus apresentou a mais baixa consistência (alfa $=0,448$ ). Pragma obteve coeficiente alfa razoável (alfa $=0,648$ ), bem como todos os seus itens. Estorge, Ágape e Mania foram as subescalas que apresentaram maior consistência (alfa de 0,898, 0,881 e 0,867, respectivamente). Dessas, apenas a questão 11 ("Nosso amor é de fato uma profunda amizade, não uma emoção misteriosa e mística."), de Estorge, não apresentou desempenho adequado.

\section{Escala de Avaliação do Relacionamento}

O nível de satisfação no relacionamento foi mais alto no grupo saudável: $85 \%$ dos indivíduos obtiveram escores acima de 20, comparado a somente $16 \%$ do grupo com amor patológico.

Todos os itens da escala distinguiram significativamente indivíduos saudáveis daqueles com amor patológico, exceto pelo item 6 (Tabela 1). Não houve diferença significativa entre os grupos quanto à compreensão dos itens (médias de compreensão entre 4,5 e 4,9). Para ambos os grupos, houve correlação negativa entre escolaridade e os itens $2(\mathrm{R}=-0,39 ; \mathrm{p}<0,05), 3(\mathrm{R}=-0,47 ; \mathrm{p}<0,01), 5(\mathrm{R}=$ $-0,44 ; \mathrm{p}<0,01), 7(\mathrm{R}=-0,34 ; \mathrm{p}<0,05)$ e o escore total $(\mathrm{R}=-0,345$; $\mathrm{p}<0,05)$. A correlação de Spearman mostrou que o item 6 não se correlaciona com os demais e os itens 4 e 5 não se correlacionam entre si. A consistência interna da escala foi de 0,85 e os itens $1,2,3$ e 5 foram os mais consistentes.

\section{Tipos de Apego do Adulto}

Houve diferenças significativas quanto ao tipo de apego entre os grupos $\left(\chi^{2}=9,25 ; p=0,01\right)$ (Tabela 1). Oitenta por cento dos indivíduos saudáveis apresentaram o tipo de apego seguro, enquanto $45 \%$ dos normais apresentaram o tipo de apego ansioso/resistente e $40 \%$, o tipo seguro. Não houve diferença significativa quanto à compreensão dos itens entre os grupos (médias de compreensão entre 4,2 e 4,7), apesar da associação significativa entre escolaridade e tipo de apego $(F=6,98 ; p<0,01)$.

\section{Discussão}

O instrumento Escalas de Atitude do Amor mostrou discriminar corretamente indivíduos saudáveis daqueles com amor patológico. Como era esperado, o grupo com amor patológico é predominantemente caracterizado por Mania (e Ágape, dada a significativa correlação observada entre esses estilos), enquanto o grupo saudável apresenta, em sua maioria, o estilo de amor Eros. No entanto, em se tratando de um instrumento de autopreenchimento, procurou-se investigar se esses conceitos sofriam influência da escolaridade sobre o endosso, descobrindo-se que apenas Eros apresentou tal correlação. Por outro lado, verificou-se, ao fazer o teste de regressão logística, que os estilos predominantes nos grupos (Mania e Eros) se mantiveram significativos quando controlados para a escolaridade.

No que diz respeito à compreensão dessa escala, ambos os grupos apresentaram bom entendimento, mas cabe observar que os indivíduos com amor patológico, embora com maior grau de instrução em relação aos saudáveis, tiveram maior dificuldade com as questões 1,2 , 4, 5 e 8. Por outro lado, não houve interferência da compreensão na pontuação dessas questões. Propõe-se que tal dificuldade de compreensão possa ser inerente ao quadro clínico, não estando relacionada à escolaridade. Segundo essa hipótese, os indivíduos com amor patológico não se identificariam com determinados conceitos que pareceriam corriqueiros para indivíduos saudáveis, por exemplo, o exprimido na questão 5 (a questão com maior problema de compreensão) que diz: "Eu acredito que o que o meu parceiro não sabe sobre mim não 
Tabela 1. Comparação entre indivíduos saudáveis e com amor patológico quanto aos dados demográficos e características do relacionamento

\begin{tabular}{|c|c|c|c|c|}
\hline Variável & Grupo AP & $\begin{array}{l}\text { Grupo } \\
\text { saudável }\end{array}$ & $\begin{array}{l}\text { Teste } \\
\text { (valor) }\end{array}$ & $P$ \\
\hline \multicolumn{5}{|l|}{ Dados demográficos } \\
\hline & $n(\%)$ & $\mathrm{n}(\%)$ & & \\
\hline Sexo & & & $X_{2}(0,009)$ & 0,925 \\
\hline Feminino & $14(74 \%)$ & $15(75 \%)$ & & \\
\hline Masculino & $5(26 \%)$ & $5(25 \%)$ & & \\
\hline Situação & & & $X^{2}(0,041)$ & 0,839 \\
\hline Com parceiro & $12(63 \%)$ & $12(60 \%)$ & & \\
\hline \multirow[t]{2}{*}{ Sem parceiro } & $7(37 \%)$ & $8(40 \%)$ & & \\
\hline & $\begin{array}{l}\text { média de } \\
\text { anos (DP) }\end{array}$ & $\begin{array}{l}\text { média de } \\
\text { anos (DP) }\end{array}$ & & \\
\hline Idade & $39,8(10,9)$ & $35,6(7,3)$ & $t(1,448)$ & 0,156 \\
\hline Escolaridade & $15,9(3,7)$ & $12,8(3,1)$ & $t(-2,946)$ & 0,006 \\
\hline \multicolumn{5}{|l|}{ Love Attitudes Scale } \\
\hline & Média (DP) & Média (DP) & & \\
\hline \multicolumn{5}{|l|}{ Estilos de Amor } \\
\hline Eros & $11,9(2,6)$ & $8,1(3,2)$ & $t(4,1)$ & $<0,001$ \\
\hline Ludus & $11,6(4,2)$ & $13,8(2,6)$ & $t(-1,9)$ & 0,069 \\
\hline Storge & $16,8(4,0)$ & $11,2(4,9)$ & $\mathrm{t}(3,9)$ & $<0,001$ \\
\hline Pragma & $15,9(3,6)$ & $14,8(3,2)$ & $t(1,1)$ & 0,299 \\
\hline Mania & $6,9(2,9)$ & $15,2(3,4)$ & $t(-8,1)$ & $<0,001$ \\
\hline Ágape & $8,9(3,8)$ & $15,1(3,8)$ & $t(-5,1)$ & $<0,001$ \\
\hline \multicolumn{5}{|l|}{ Relationship Assesment Scale } \\
\hline & Média (DP) & Média (DP) & & \\
\hline \multicolumn{5}{|l|}{ Item } \\
\hline 1 Atendimento das necessidades & $2,0(1,1)$ & $3,7(1,1)$ & $U(56,50)$ & $<0,001$ \\
\hline 2 Satisfação no relacionamento & $1,7(0,7)$ & $3,6(1,1)$ & $U(38,00)$ & $<0,001$ \\
\hline $\begin{array}{l}3 \text { Comparação com os demais } \\
\text { relacionamentos }\end{array}$ & $2,1(0,9)$ & $4,0(0,9)$ & $U(33,50)$ & $<0,001$ \\
\hline 4 Arrependimento & $2,7(1,1)$ & $3,8(1,4)$ & $U(107,50)$ & 0,017 \\
\hline $\begin{array}{l}5 \text { Preenchimento das } \\
\text { expectativas }\end{array}$ & $1,6(0,7)$ & $3,5(1,1)$ & $U(33,50)$ & $<0,001$ \\
\hline 6 Intensidade do amor & $4,4(0,7)$ & $3,8(1,2)$ & $U(126,50)$ & 0,057 \\
\hline 7 Problemas & $2,1(1,3)$ & $4,5(0,9)$ & $U(31,00)$ & $<0,001$ \\
\hline Pontuação total & $16,6(3,2)$ & $26,8(5,6)$ & $t(6,84)$ & $<0,001$ \\
\hline \multicolumn{5}{|l|}{ Adult attachment types } \\
\hline & $n(\%)$ & $n(\%)$ & & \\
\hline \multicolumn{5}{|l|}{ Tipo de apego } \\
\hline Seguro & $8(42 \%)$ & $16(80 \%)$ & $x^{2}(4,419)$ & 0,036 \\
\hline Ansioso/com evitação & $2(11 \%)$ & $3(15 \%)$ & $x^{2}(0,000)$ & 1,000 \\
\hline Ansioso/resistente & $9(47 \%)$ & $1(5 \%)$ & $x^{2}(7,086)$ & 0,008 \\
\hline
\end{tabular}

p: nível de significância; $x^{2}$ : qui-quadrado; $t=t$ de Student; U: Mann-Whitney; DP: desvio-padrão.

vai machucá-lo.” Indivíduos que sofrem de amor patológico podem não entender esse conceito, porque acreditam que deva haver uma espécie de "fusão" entre os parceiros, na qual não é permitido haver segredos. Quanto à consistência interna da escala, Estorge, Mania e Ágape mostraram-se os conceitos mais sólidos, fato que se sobrepõe à constatação anterior de que são justamente esses estilos, exceto por Estorge, que, de fato, diferenciaram os indivíduos com amor patológico dos saudáveis. Eros demonstrou consistência razoável, com exceção do item 4 ("Meu parceiro se encaixa nos meus padrões ideais de beleza física."). Visto que esse estilo de amor tende a relacionar-se diretamente à atração física entre os parceiros, esse item poderia ser reformulado com algo menos restritivo do que "padrões ideais". Ludus obteve baixa consistência em dois de seus itens, 5 ("Eu acredito que aquilo que o meu parceiro não sabe sobre mim não vai machucá-lo.") e 8 ("Eu gosto do 'jogo da sedução' tanto com meu parceiro quanto com outros."). A baixa consistência da questão 8 possivelmente se deve à expressão “jogo da sedução", que já havia gerado dúvidas anteriormente na etapa da revisão das escalas feita pelos profissionais da saúde. Pragma obteve alfa razoável e todos os itens contribuíram de forma significativa. Nesse caso, o conceito poderia ser melhorado aumentando o número de itens relacionados a esse estilo.
No que diz respeito à Escala de Avaliação do Relacionamento, como esperado, confirmou-se que os voluntários saudáveis mostraram-se mais satisfeitos em seus relacionamentos do que os indivíduos com amor patológico. Os itens da escala demonstraram discriminar corretamente os grupos, com exceção do item 6, provavelmente pelo fato de esse item exprimir um conceito não necessariamente relacionado às demais questões, pois refere-se à intensidade do amor pelo parceiro ("Quanto você ama o seu parceiro?"). Indivíduos com amor patológico estão frequentemente insatisfeitos na relação e, ainda assim, referem amar com grande intensidade. Esse item deveria ser pontuado separadamente das demais questões, de maneira a não interferir no escore total da escala. Quanto à compreensão dos itens que compõem a escala, tanto indivíduos com amor patológico quanto voluntários saudáveis obtiveram entendimento muito bom. A correlação negativa entre os itens 2, 3, 5 e 7 e o escore e a escolaridade mostrou que, nesses itens, o grupo com amor patológico (maior escolaridade) pontua menos (menor satisfação) do que o grupo de voluntários saudáveis, influenciando diretamente no escore total. Além disso, os itens 1, 2, 3, 5 e 7 são os mais consistentes da escala, enquanto os itens 4 e 6 não contribuem para a sua solidez. A baixa consistência do item 6 pode ser explicada pelo fato, já mencionado, de esse item não expressar um conceito que caracterize necessariamente satisfação no relacionamento, principalmente no caso dos indivíduos com amor patológico. Já a baixa consistência do item 4 ("Com que frequência você desejaria não ter começado esse relacionamento?") também pode ser explicada por motivo semelhante, já que os indivíduos com amor patológico, em sua grande maioria, não se arrependem de ter iniciado um relacionamento com o parceiro, mesmo considerando que ele os faz sofrer. Ao montar-se um modelo adaptado da Escala de Avaliação do Relacionamento, somente com as questões de maior consistência (1, 2, 3, 5 e 7), obteve-se maior significância no que diz respeito às diferenças entre indivíduos com amor patológico e saudáveis, demonstrando que esses itens são os que diferenciam de maneira mais eficiente o conceito de amor patológico.

Quanto ao instrumento Tipos de Apego do Adulto, observou-se que os indivíduos com amor patológico são, mais frequentemente, caracterizados pelo tipo de apego ansioso/ambivalente. No entanto, uma proporção razoável de indivíduos com amor patológico apresentou o tipo de apego seguro. A hipótese é de que isso tenha ocorrido pelo fato de a escala ser formada por apenas um item para representar cada um dos tipos de apego. Para melhorar a estrutura psicométrica da escala, o ideal seria aumentar o número de itens para cada categoria de apego, de maneira a formar escores para cada uma delas, visando discriminar o maior número possível de pessoas. A associação verificada entre escolaridade e tipo de apego se explica pelo fato de que o grupo saudável, que possui menor escolaridade, foi predominantemente caracterizado pelo tipo de apego seguro $(80 \%)$, o que não indica, a princípio, uma relação direta entre escolaridade e tipo de apego. Apesar disso, a escala mostrou-se de fácil entendimento, não havendo diferenças de compreensão entre indivíduos saudáveis e aqueles com amor patológico, ou entre indivíduos com diferentes tipos de apego.

Para o presente estudo, no que diz respeito às características psicométricas das escalas, não foi possível a comparação dos instrumentos traduzidos e adaptados com os instrumentos originais, visto que nem mesmo em seu país de origem esses instrumentos foram submetidos a estudos anteriores de validade e confiabilidade.

Apesar do número limitado de indivíduos da amostra, as versões traduzidas e adaptadas neste trabalho apresentaram validade de critério e validade de construção satisfatórias, visto que discriminam significativamente indivíduos com amor patológico de indivíduos saudáveis, assim como são capazes de mensurar corretamente a satisfação no relacionamento, o tipo de apego e o estilo de amor do indivíduo. Além disso, apresentaram boa aplicação e entendimento nas amostras avaliadas.

Tais achados justificam a utilização desses instrumentos validados para o português em pesquisas de investigação do amor patológico, ou outras que necessitem averiguar características pessoais relacionadas ao amor e aos relacionamentos.

Estudos posteriores mais aprofundados deverão determinar as características psicométricas desses instrumentos para a população brasileira quanto à confiabilidade teste/reteste e a confiabilidade entre avaliadores. Novos testes com amostras mais homogêneas quanto à escolaridade também poderão apresentar resultados mais consistentes. 


\section{Referências}

1. Simon J. Love: addiction or road to self-realization, a second look. Am J Psychoanal. 1982;42(3):253-63.

2. Fisher M. Personal Love. London: Duckworth; 1990

3. Sophia EC, Tavares H, Zilberman ML. Pathological love: is it a new psychiatric disorder? Rev Bras Psiquiatr. 2007;29(1):55-62

4. Norwood R. Women who love too much. Los Angeles, CA: Jeremy PTarcher Press; 1985.

5. Wang Z, Aragona BJ. Neurochemical regulation of pair bonding in male prairie voles. Physiol Behav. 2004;15:83(2):319-28.

6. Donnellan MB, Larsen-Riffe D, Conger RD. Personality, family, history, and competence in early adult romantic relationships. J Pers Soc Psychol. 2005;88(3):562-76.

7. Timmreck TC. Overcoming the loss of a love: preventing love addiction and promoting emotional health. Psychol Rep. 1990;66(2):515-28.

8. Gjerde PF, Onishi M, Carlson KS. Personality characteristics associated with romantic attachment: a comparison of interview and self-report methodologies. Pers Soc Psychol Bull. 2004;30(11):1402-15.

9. Nelson ES, Hill-Barlow D, Benedict JO. Addiction versus intimacy as related to sexual involvement in a relationship. J Sex Marital Ther. 1994;20(1):35-45.

\section{ANEXO 1}

\section{ESCALA DE ATITUDES DO AMOR}

A seguir estão listadas várias afirmações que mostram diferentes atitudes sobre o amo Para cada afirmação, preencha com sua resposta a Folha de Respostas que indica o quanto você concorda com aquela afirmação ou discorda dela. Os itens referem-se a um relacionamento amoroso específico. Sempre que possível, responda às questões tendo o seu(sua) parceiro(a) atual em mente. Se você não tiver um(a) parceiro(a) no momento, responda à questão tendo em mente seu(sua) parceiro(a) mais recente. Se você nunca se apaixonou por alguém, responda em termos de como você acha que seriam as suas respostas.

Para cada afirmação, responda:

$A=$ Concordo totalmente com a afirmação.

$\mathrm{B}=$ Concordo em grande parte com a afirmação.

C = Neutro - nem concordo nem discordo.

$\mathrm{D}=$ Discordo em grande parte da afirmação.

$\mathrm{E}=$ Discordo totalmente da afirmação.

1. Meu(minha) parceiro(a) e eu temos a "química" certa entre nós

2. Eu sinto que meu(minha) parceiro(a) e eu fomos feitos um para o outro.

3. Meu(minha) parceiro(a) e eu nos entendemos muito bem.

4. Meu(minha) parceiro(a) se encaixa nos meus padrões ideais de beleza física.

5. Eu acredito que aquilo que o meu(minha) parceiro(a) não sabe sobre mim não vai machucá-lo(a).

6. Algumas vezes, eu tive que evitar que o meu(minha) parceiro(a) descobrisse sobre outros(as) parceiros(as).

7. Meu(minha) parceiro(a) ficaria triste se soubesse de algumas das coisas que eu fiz com outros(as) parceiros(as).

8. Eu gosto do "jogo da sedução" tanto com meu(minha) parceiro(a) quanto com outros(as).

9. Nosso amor é do melhor tipo, pois surgiu de uma longa amizade.

10. Com o tempo nossa amizade se transformou gradualmente em amor.

11. Nosso amor é de fato uma profunda amizade, não uma emoção misteriosa e mística.

12. Nosso relacionamento é o mais satisfatório, pois se desenvolveu a partir de uma boa amizade

13. 0 que mais pesou na escolha do meu(minha) parceiro(a) foi como ele(a) seria visto(a) pela minha família.

14. Um fator importante na escolha do meu(minha) parceiro(a) foi se ele(a) seria ou não um bom pai ou uma boa mãe.

15. Um fator considerado na escolha do meu(minha) parceiro(a) foi como ele(a) interferiria na minha carreira.

16. Antes de me envolver muito com meu(minha) parceiro(a), eu tentei perceber se seu traços hereditários seriam compatíveis com os meus, em caso de termos um filho.

17. Quando meu(minha) parceiro(a) não presta atenção em mim, eu fico muito mal.

18. Desde que eu me apaixonei pelo meu(minha) parceiro(a), eu tenho tido problemas para me concentrar em outras coisas.

19. Eu não consigo relaxar se eu suspeitar que meu(minha) parceiro(a) está com outra pessoa.

20. Se meu(minha) parceiro(a) me ignora por algum tempo, eu às vezes faço coisas estúpidas para atrair novamente sua atenção.

21. Eu prefiro sofrer eu mesma(o) a deixar meu(minha) parceiro(a) sofrer.

22. Eu não consigo ficar feliz a menos que eu coloque a felicidade do meu(minha) parceiro(a) antes da minha própria.

23. Eu geralmente me disponho a sacrificar meus próprios desejos em função dos desejos do meu(minha) parceiro(a).

24. Eu aguentaria passar por qualquer coisa pelo bem do meu(minha) parceiro(a)
10. Bogerts B. Delusional jealousy and obsessive love-causes and forms MMW Fortschr Med. 2005;147(6):26-9.

11. Moss E. Treating the love-sick patient. Isr J Psychiatry Relat Sci. 1995;32(3):167-73

12. Menezes, PR. Validade e confiabilidade das escalas de avaliação em psiquiatria. Rev Psiq Clín. 1998;25(5):214-6.

13. Hendrick C, Hendrick SS, Dicke A. The Love Attitude Scale: short form J Soc Pers Rel. 1998;15:147-59.

14. Lee JA. A typology of styles of loving. Pers Soc Psychol Bull. 1977;3:173-82.

15. Hendrick SS. A generic measure of relationship satisfaction. J Marriage Fam. 1988;50:93-8.

16. Hazan C, Shaver P. Romantic love conceptualized as an attachment process. J Pers Soc Psychol. 1987;52:511-24.

17. Bowlby J. Attachment and Loss: vol. 1. Attachment. New York: Basic Books; 1969

18. Ainsworth MDS, Blehar MC, Waters E, Wall S. Patterns of attachment: a psychological study of the strange situation. Hillside (NJ): Erlbaum; 1978

19. Blay SL. Comentários do debatedor: escalas de avaliação utilizadas em múltiplos quadros. Rev Psiq Clín. 1999;26(2).

20. Del-Ben CM, Rodrigues CR, Zuardi AW. Reliability of the Portuguese version of the structured clinical interview for DSM-III-R (SCID) in a Brazilian sample of psychiatry outpatients. Braz J Med Biol Res. 1996;29(12):1675-82.

\section{ANEXO 2}

ESCALA DE AVALIAÇÃO DE RELACIONAMENTO

\begin{tabular}{|c|c|c|}
\hline \multicolumn{3}{|c|}{ 1. Quanto o(a) seu(sua) parceiro(a) preenche suas necessidades? } \\
\hline A $\quad B$ & C D & E \\
\hline Pouco & Na média & Muitíssimo \\
\hline \multicolumn{3}{|c|}{ 2. Em geral, quanto você está satisfeita(o) com o seu relacionamento? } \\
\hline A $\quad B$ & C D & E \\
\hline Insatisfeito & Na média & Extremamente satisfeito \\
\hline
\end{tabular}

3. Em comparação com a maioria das pessoas, quanto o seu relacionamento bom?

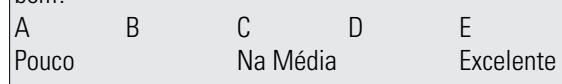

4. Com que frequência você desejaria não ter começado esse relacionamento?

$\begin{array}{lllll}\text { A } & B & C & D & E \\ \text { Nunca } & & \text { Às vezes } & & \text { Frequentemente }\end{array}$

5. Em que medida o seu relacionamento tem satisfeito suas expectativas iniciais?

$\begin{array}{lllll}\text { A } & B & C & D & \text { E } \\ \text { Pouco } & & \text { Na média } & & \text { Completamente }\end{array}$

6. Quanto você ama o(a) seu(sua) parceiro(a)?

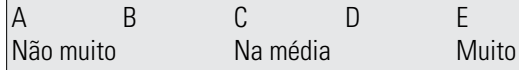

7. 0 quanto o seu relacionamento é problemático?

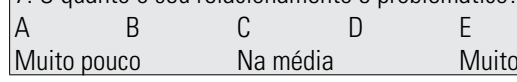

\section{ANEXO 3}

TIPOS DE APEGO ADULTO

Pergunta: Que situação a seguir melhor descreve os seus sentimentos em relação às outras pessoas?

1) Eu acho relativamente fácil me aproximar de outras pessoas e me sinto à vontade em confiar nelas e deixar que elas confiem em mim. Eu geralmente não me preocupo em ser abandonado ou que alguém se torne íntimo de mim. 2) Eu me sinto pouco à vontade em ficar muito próximo de outras pessoas: acho difícil confiar nelas completamente ou permitir que eu dependa delas. Eu fico nervoso quando alguém se aproxima muito e, com frequência, meus(minhas) parceiros(as) querem que eu seja mais íntimo do que eu consigo ser. 3) Eu sinto que os outros relutam em se aproximar tanto quanto eu gostaria. Frequentemente eu acho que o(a) meu(minha) parceiro(a) não me ama de verdade, ou que não vai querer ficar comigo. Eu quero me unir completamente com a outra pessoa e esse desejo às vezes afugenta as pessoas. 